PHYSICAL SCIENCES

\title{
S-PLUS: LEnticular Galaxies in Stripe 82 (LEGS82)
}

\author{
ARIANNA CORTESI, KANAK SAHA, FABRICIO FERRARI, GEFERSON LUCATELLI, \\ CLAUDIA M. DE OLIVEIRA, SURAJ DHIWAR, CLECIO R. BOM \& LUCIANA OLIVIA DIAS
}

\begin{abstract}
This work is a Brazilian-Indian collaboration. It aims at investigating the structural properties of Lenticular galaxies in the Stripe 82 using a combination of S-PLUS (Southern Photometric Local Universe Survey) and SDSS data. S-PLUS is a novel optical multi-wavelength survey which will cover nearly 8000 square degrees of the Southern hemisphere in the next years and the first data release covers the Stripe 82 area. The morphological classification and study of the galaxies' stellar population will be performed combining the Bayesian Spectral type (from BPZ) and Morfometryka (MFMTK) parameters. BPZ and MFMTK are two complementary techniques, since the first one determines the most likely stellar population of a galaxy, in order to obtain its photometric redshift (phot-z), and the second one recovers non-parametric morphological quantities, such as asymmetries and concentration. The combination of the two methods allows us to explore the correlation between galaxies shapes (smooth, with spiral arms, etc.) and their stellar contents (old or young population). The preliminary results, presented in this work, show how this novel data set opens a new window on our understanding of the nearby universe.
\end{abstract}

Key words: galaxy morphology, galaxy evolution, lenticular galaxies, galaxy stellar population, fundamental parameters.

\section{INTRODUCTION}

Lenticular galaxies have been largely studied, since when they have been first classified by Edwin Hubble (Hubble 1936), as the missing link between elliptical and spiral galaxies. Sos or lenticular galaxies present, in fact, an hybrid structure, showing a prominent disk, but with no signs of spiral arms. The presence of the disk suggests that their kinematics is rotationally supported (Cortesi et al. 2013b,a), as found for spiral galaxies Merrett et al. (2003). Elliptical galaxies, on the contrary, are pressure supported systems, as a result of their formation histories, which comprise a sequence of major and minor mergers (Bournaud et al. 2005). Moreover, lenticular galaxies are more frequently found in denser regions of galaxy clusters (Dressler 1980) and their frequency increases towards low redshifts (Dressler et al. 1997). Considering that this is an opposite trend to that of spiral galaxies, it has been first hypothesised that So galaxies may be the end product of spiral galaxies whose gas was gently stripped or consumed. Such evolutionary path would require the interplay between the galaxy and the surrounding environment to cease star formation and remove the spiral arms. Yet, field lenticular galaxies exist. At the present moment, several mechanisms have been pointed out as possible ways to create lenticular galaxies: minor mergers (Bournaud et al. 2005), major mergers under specific initial conditions (Eliche-Moral 
et al. 2018), AGN feedback or/and secular evolution (Mishra et al. 2018), and it is becoming more popular the idea that the so class is actually a compilation of objects that formed through very different paths and just happen to have similar appearances. Part of the problem stands on the difficulty of visually identifying lenticular galaxies. In fact, there is not a consensus on the morphological characteristics of lenticular galaxies and they can, in general, be easily misclassified due to the vague appearence of spiral arms, and not trivial determination of the presence of a disk, from a purely photometric approach. Large spectroscopic surveys, on the other side, often covers only the central part of a galaxy, i.e. the region where the bulge dominates, again making it hard to separate elliptical galaxies, from lenticular galaxies with large bulge-over-total light ratio. Multi-wavelength surveys (as S-PLUS (Mendes de Oliveira et al. 2019)) open a new window on galaxy classification, combining the possibility of studying the variation of morphological parameters with wavelengths and recovering the galaxy stellar population. We present preliminary results of this approach, using the S-PLUS first data release ${ }^{1}$, and we describe new methods to identify lenticular galaxies.

\section{DATA AND METHODS}

S-PLUS (Southern Photometric Local Universe Survey) is an astronomical facility in Chile (Cerro Pachon), dedicated to mapping the observable sky in 7 narrow-band filters and 5 broad-band (ugriz) filters in the optical region. The $0.86 \mathrm{~m}$ mirror of the T80-South telescope, combined with a field of view of 1.4 square degrees and an 85 Mega-pixel camera, is producing high-quality images and a unique spectral resolution for millions of objects over several thousand square degrees. Together with its twin observatory in the northern hemisphere, the T80-North, its sister survey J-PLUS, will cast the first light on a multi-colour Universe, on about half of the extragalactic sky. This multi-purpose astrophysical survey in the southern hemisphere started in 2016. During the next 3-4 years it will observe more than 8,000 square degrees $(1 / 5$ of the whole sky), covering the entire visible region of the electromagnetic spectrum (3500 $\mathrm{A}$ to 10,000 A). Figure 1 and 2 show the area covered in the sky and the Javalambre filter system used by S-PLUS.

Galaxy morphologies vary with wavelength, since different stellar populations present different colours. In general, star forming galaxies have a lower bulge-to-total $(B / T)$ light ratio than quiescent ones (Morselli et al. 2017). We use Morfometryka (Ferrari et al. 2015) (MFMTK) to compute galaxies' non-parametric morphometric parameters, in particular we recover the concentration ( $\mathrm{C}_{1}$ ) and the entropy $(\mathrm{H})$. The concentration is defined as the ratio of the radii containing some fraction of the total light inside the Petrosian Region (2Rp):

$$
C_{1}=\log \left(\frac{R_{80}}{R_{20}}\right),
$$

where $R_{f}$ is the radius that contains a fraction of $f \%$. Entropy is defined as:

$$
H(I)=-\sum_{i}^{n} p\left(I_{i}\right) \ln \left[p\left(I_{i}\right)\right] / H_{\max }
$$

where $p$ is the probability of the occurence of the intensity $I_{i}$ and $H_{\max }$ is the maximum entropy, which is for an homogeneous distribution (e.g. $p=1 / n$ ). For the early type galaxies the concentration is nearly constant with wavelength, while it increases for redder wavelengths, in the case of the spiral galaxies. Another feature is that homogeneous systems

\footnotetext{
${ }^{1}$ https://datalab.noao.edu/splus/
} 


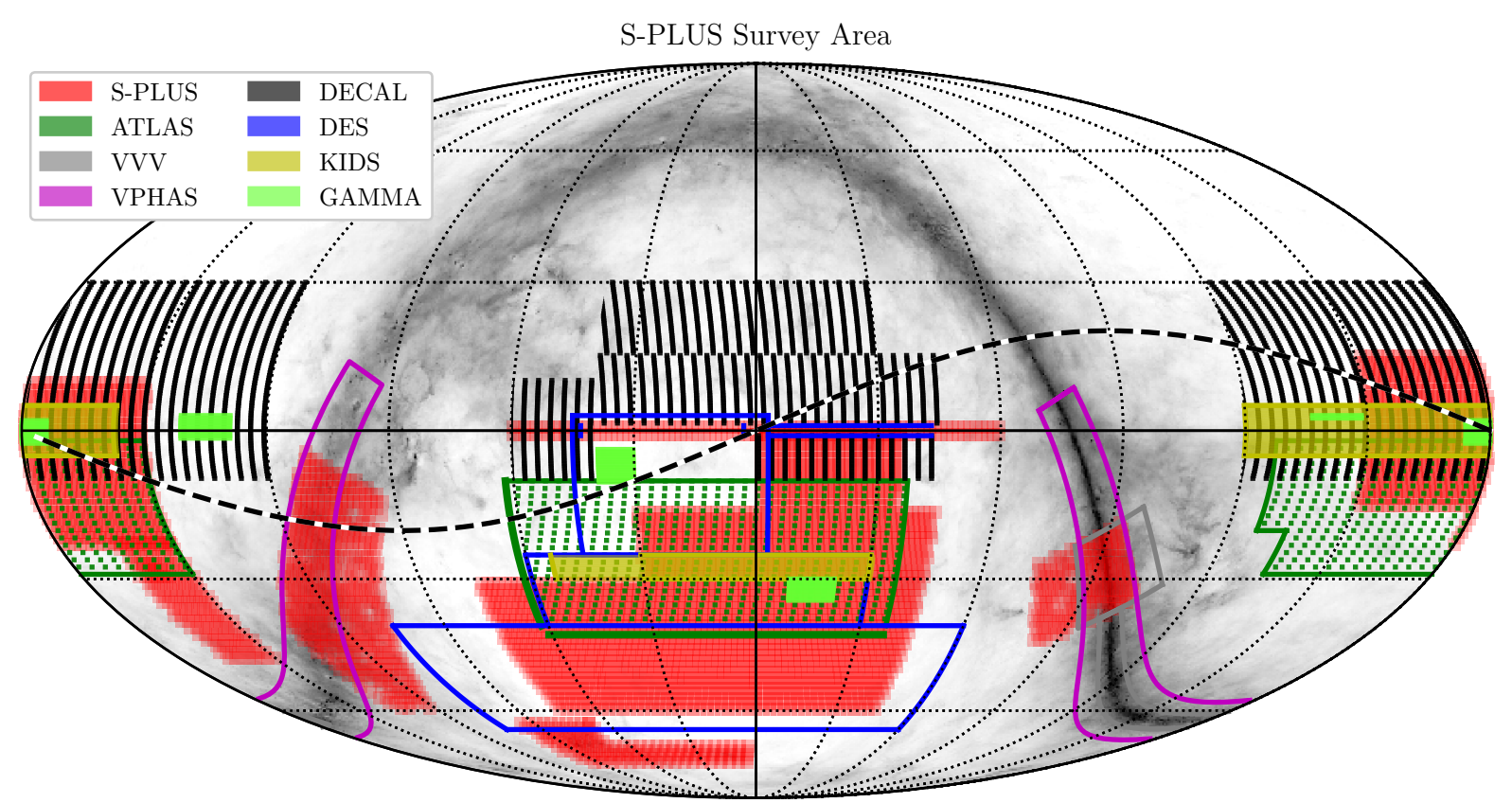

Figure 1. Diagram showing some of the most important optical and near-infrared surveys in the Southern Hemisphere (we omit the surveys SkyMapper, Gaia and LSST that cover the entire hemisphere or sky). For the optical surveys: ATLAS is shown in hatched green, VPHAS+ is the pink rectangular contour over the Bulge and Disk of the Galaxy, DECAL is in hatched black, DES is shown in blue, KiDS in yellow, and GAMA in bright green. The only near-infrared survey displayed is VISTA-VVV, a rectangle with light gray contours over the Galactic disk. Dark red shows the area covered by S-PLUS. The broken black line represents the ecliptic. The figure is taken from (Mendes de Oliveira et al. 2019).

(with less concentrated light distributions, such as disks or extended galaxies) have higher entropies than systems with more concentrated light distributions (ellipticals, bulge dominated, compacted, etc.). Finally, we use the Bayesian Photometric redshifts (BPZ) (Molino et al. 2014) method to obtain the galaxy bayesian spectral type, by identifying the galaxy template model that optimises the redshift determination, for more details see Molino et al. (2020).

\section{RESULTS}

The left panel of Figure 3 shows the distribution of the galaxies in the $\mathrm{H}-\mathrm{C} 1$ plane, colour coded according to their morphological type (Nair \& Abraham 2010). The T-Type parameter value is lower than -3 for elliptical galaxies, between -3 to 0 for lenticular galaxies, and higher than 0 for spiral and irregular galaxies. This plot clearly shows that only using these two MFMTK (Ferrari et al. 2015) parameters we are able to separate galaxies into different classes. Combining these parameters with other MFMTK outputs (such as asymmetries, clumpiness, Gini parameter, spirality ..) and the curvature of the brightness profile of the galaxies (Lucatelli \& Ferrari 2019) at every wavelengths, we are able to identify a bona-fide catalogue of lenticular galaxies for the S-PLUS DR1 data release (Lucatelli et al. in prep).

The right panel of Figure 3 presents the same $H-C 1$ relation, but this time the objects are colour coded according to their Bayesian Spectral Type (Tb), as defined in Molino et al. (2020). The galaxies' templates are defined as follows: from $\mathrm{T}_{1}$ to $\mathrm{T}_{4}$ is a spectral energy distribution (SED) typical of elliptical galaxies, 


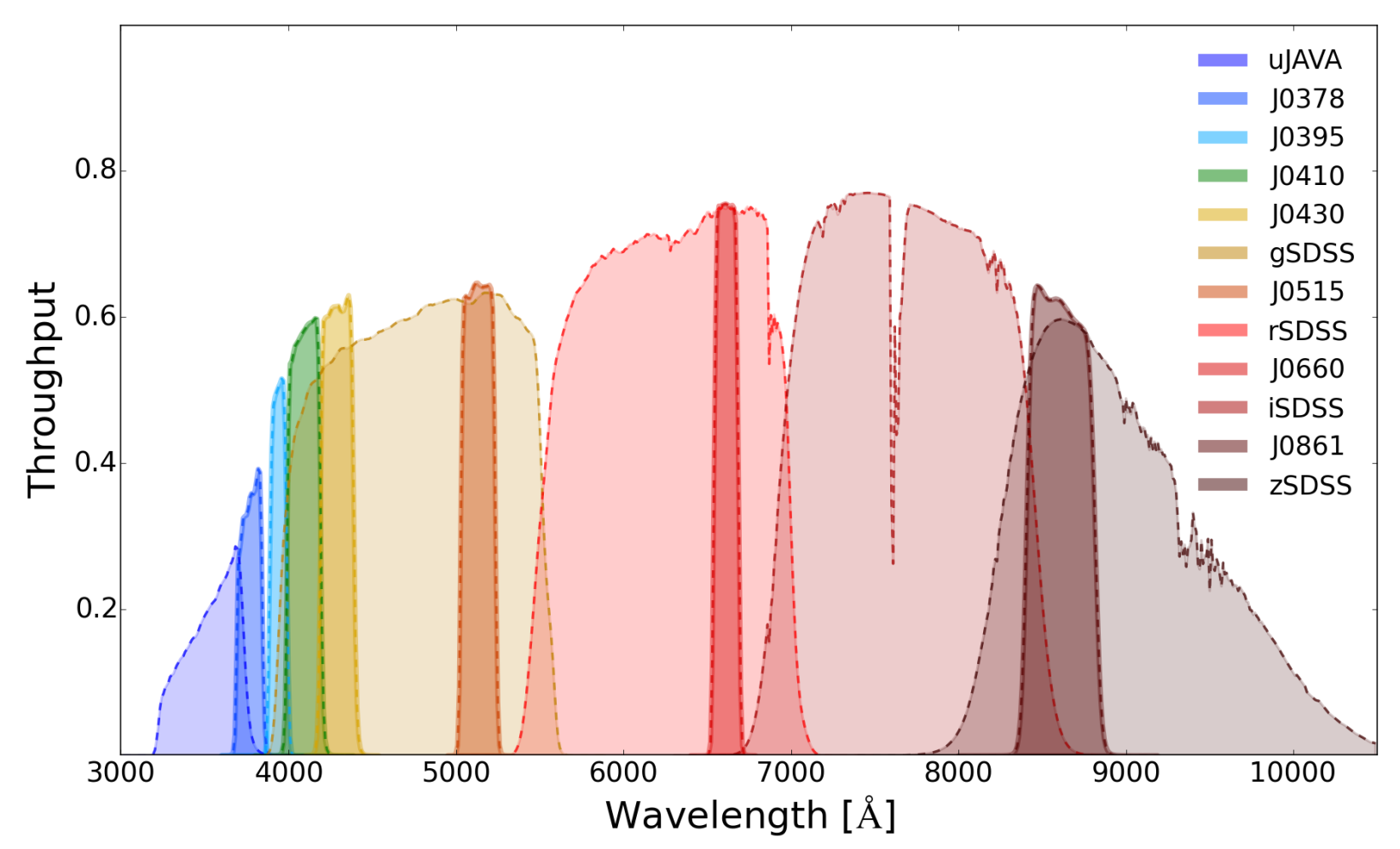

Figure 2. The Javalambre 12-filter system used by S-PLUS. The y-axis shows the total efficiency of the filters, obtained through the multiplication of the average transmission curves, the CCD efficiency, and the mirror reflectivity curves. Different filters are coloured according to the labels on the right-side panel. The figure is taken from Mendes de Oliveira et al. (2019).
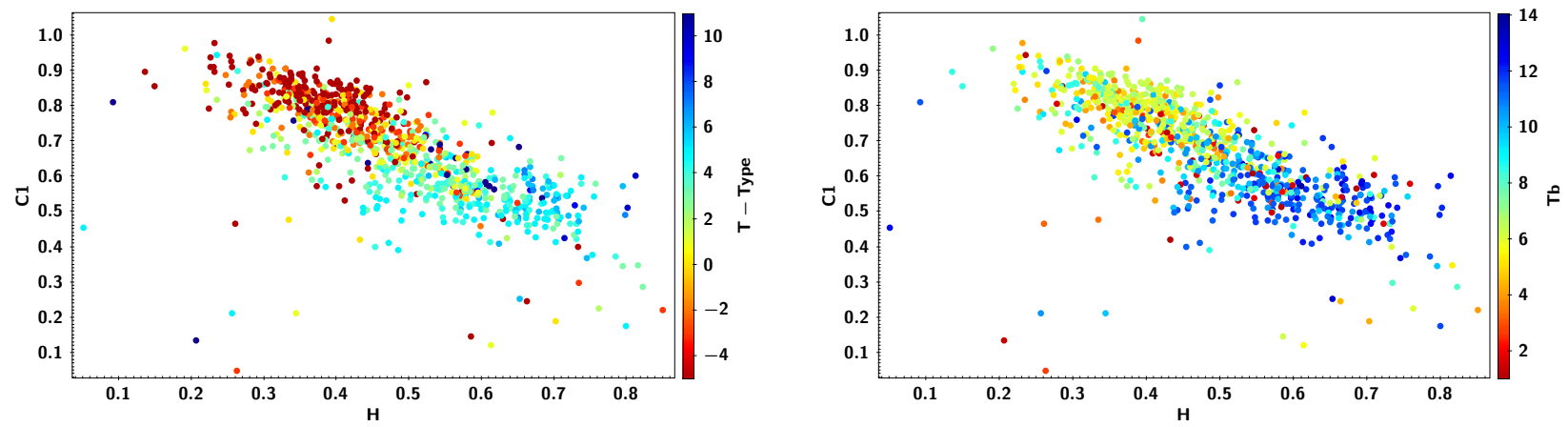

Figure 3. Entropy versus concentration parameters, estimated using MFMTK, for a sample of 925 galaxies, colour coded according to their morphological T-Type, left panel, and according to their bayesian spectral templates, right panel. See text for more details. 
T5 is for ESo galaxies, from T6 to T14 are spiral galaxies templates (barred and un-barred). Again, the morphometric parameters allow us to divide galaxies into passive (early-type galaxies) and star forming (late-type galaxies). Interestingly, several T1 galaxies lie in the part of the plot populated by spiral galaxies, and some galaxies characterised by a spiral-like galaxy templates fall in the upper area of the plot, where we expect to find early type galaxies. This finding shows that galaxies' morphology and stellar content are not always as expected: the universe present blue ellipticals and red spirals (Strateva et al. 2001). S-PLUS data set allows to recover both these quantities and map the galaxy distribution in the local universe.

\section{CONCLUSIONS}

In this work we present preliminary results of the study of galaxy morphology and stellar population properties obtained using data of the S-PLUS survey. We show how the parameters recovered with MFMTK allow to perform a galaxy morphological classification and we suggest a way to identify so galaxies, combining such parameters with a novel method based on the study of the curvature of the galaxies' surface light profile (Lucatelli \& Ferrari 2019). The S-PLUS data set, given its unique combination of narrow and broad band filters, allows the estimation of the galaxy most probable stellar template, together with the definition of its morphometric parameters. This study extends from observational astronomy, to theory and computational analysis; it comprises researchers from Brazil and India and it is open to a world-wide collaboration. With its $0.86 \mathrm{~cm}$ diameter, S-PLUS proves the importance of small telescopes in the advance of astronomical knowledge. Several other small telescopes exist in the BRICS countries. We conclude encouraging a dialogue among these telescope users, sharing technical and scientific knowledge.

\section{Acknowledgments}

AC acknowledges support from PNPD/CAPES.

\section{REFERENCES}

BOURNAUD F, JOG CJ \& COMBES F. 2005. Galaxy mergers with various mass ratios: Properties of remnants. A\&A 437: 69-85. doi:10.1051/0004-6361:20042036.

CORTESI A ET AL. 2013a. Planetary Nebula Spectrograph survey of So galaxy kinematics - II. Clues to the origins of So galaxies. MNRAS 432(2): 1010-1020. doi:10.1093/mnras/stt529.

CORTESI A ET AL. 2013b. The Planetary Nebula Spectrograph survey of So galaxy kinematics. Data and overview. A\&A 549: A115. doi:10.1051/00046361/201220306.

DRESSLER A. 1980. Galaxy morphology in rich clusters Implications for the formation and evolution of galaxies. ApJ 236: 351-365. doi:10.1086/157753.

DRESSLER A, OEMLER JR A, COUCH WJ, SMAIL I, ELLIS RS, BARGER A, BUTCHER H, POGGIANTI BM \& SHARPLES RM. 1997. Evolution since $Z=0.5$ of the Morphology-Density Relation for Clusters of Galaxies. ApJ 490: 577-+. doi:10.1086/304890.

ELICHE-MORAL MC, RODRIGUEZ-PÉREZ C, BORLAFF A, QUEREJETA M \& TAPIA T. 2018. Formation of So galaxies through mergers. Morphological properties: tidal relics, lenses, ovals, and other inner components. A\&A 617: A113. doi:10.1051/0004-6361/201832911.

FERRARI F, DE CARVALHO RR \& TREVISAN M. 2015. Morfometryka-A New Way of Establishing Morphological Classification of Galaxies. ApJ 814(1): 55. doi:10.1088/0004$637 X / 814 / 1 / 55$

HUBBLE EP. 1936. Realm of the Nebulae. New Haven: Yale University Press.

LUCATELLI G \& FERRARI F. 2019. Galaxy structural analysis with the curvature of the brightness profile. MNRAS 489(1): 1161-1180. doi:10.1093/mnras/stz2154.

MENDES DE OLIVEIRA C ET AL. 2019. The Southern Photometric Local Universe Survey (S-PLUS): improved SEDS, morphologies, and redshifts with 12 optical filters. MNRAS 489(1): 241-267. doi:10.1093/mnras/stz1985. 
MERRETT HR ET AL. 2003. Tracing the star stream through M31 using planetary nebula kinematics. MNRAS 346: L62-L66. doi:10.1111/j.1365-2966.2003.07367.X.

MISHRA PK, WADADEKAR Y \& BARWAY S. 2018. Why are classical bulges more common in So galaxies than in spiral galaxies? MNRAS 478(1): 351-358. doi:10.1093/mnras/sty1107.

MOLINO A ET AL. 2014. The ALHAMBRA Survey: Bayesian photometric redshifts with 23 bands for 3 deg. MNRAS 441(4): 2891-2922. doi:10.1093/mnras/stu387.

MOLINO A ET AL. 2020. Assessing the photometric redshift precision of the S-PLUS survey: the Stripe-82 as a test-case. MNRAS doi:10.1093/mnras/staa1586.

MORSELLI L, POPESSO P, ERFANIANFAR G \& CONCAS A. 2017. Bulges and discs in the local Universe. Linking the galaxy structure to star formation activity. A\&A 597: A97. doi:10.1051/0004-6361/201629409.

NAIR PB \& ABRAHAM RG. 2010. A Catalog of Detailed Visual Morphological Classifications for 14,034 Galaxies in the Sloan Digital Sky Survey. ApJS 186(2): 427-456. doi:10.1088/0067-0049/186/2/427.

STRATEVA I ET AL. 2001. Color Separation of Galaxy Types in the Sloan Digital Sky Survey Imaging Data. AJ 122(4): 1861-1874. doi:10.1086/323301.

\section{How to cite}

CORTESI A, SAHA K, FERRARI F, LUCATELLI G, OLIVEIRA CM, DHIWAR S, BOM CR \& DIAS LO. 2021. S-PLUS: LEnticular Galaxies in Stripe 82 (LEGS82). An Acad Bras Cienc 93: e2O200989. DOI 10.1590/0001-3765202120200989.

Manuscript received on June 24, 2020;

accepted for publication on August 30, 2020

\section{ARIANNA CORTESI ${ }^{1}$}

https://orcid.org/0000-0002-0620-136

KANAK SAHA ${ }^{2}$

https://orcid.org/0000-0002-8768-9298

FABRICIO FERRARI ${ }^{3}$

https://orcid.org/0000-0002-0056-1970

GEFERSON LUCATELLI 3

https://orcid.org/0000-0002-2410-1776

CLAUDIA M. DE OLIVEIRA4

https://orcid.org/0000-0002-5267-9065

\section{SURAJ DHIWAR 5}

https://orcid.org/0000-0001-8650-205X

CLECIO R. BOM 6

https://orcid.org/0000-0003-4383-2969

LUCIANA OLIVIA DIAS 6

https://orcid.org/0000-0002-7626-231X
${ }^{1}$ Observatório do Valongo (OV), Ladeira do Pedro Antônio 43, Centro, 20080-090 Rio de Janeiro, RJ, Brazil

${ }^{2}$ Inter-University Centre for Astronomy and Astrophysics (IUCAA), Post Bag 4, Ganeshkhind, Pune, Maharashtra 411007, India ${ }^{3}$ Universidade Federal do Rio Grande (FURG), Instituto de Matemática Estatística e Física (IMEF), Av. Itália, km 8, Bairro Carreiros, 96203-900 Rio Grande, RS, Brazil

4Departamento de Astronomia, Instituto de Astronomia, Geofísica e Ciências Atmosféricas da USP, Cidade Universitária, 05508-900 São Paulo, SP, Brazil

${ }^{5}$ Inter-University Centre for Astronomy and Astrophysics (IUCAA), Post Bag 4, Ganeshkhind, Pune, Maharashtra, 411007, India ${ }^{6}$ Centro Brasileiro de Pesquisas Físicas (CBPF), Rua Dr. Xavier Sigaud 150, Urca, 22290-180 Rio de Janeiro, RJ, Brazil

\section{Correspondence to: Arianna Cortesi}

E-mail:aricorte@astro.ufrj.br

\section{Authors contributions}

A.C. developed the idea of the paper, wrote the text and prepared fig. 3 and fig. 4. K.S. developed the idea and defined the name of the project. F.F. and G.L. run MFMTK on the galaxy sample, helped understanding the results and writing the paper. C.M. PI of S-PLUS, helped understanding the results and writing the paper, provided fig. 1 and fig. 2. S.D. and L.O.D. team member, helped developing the project. C.B. helped understanding the results and writing the paper.

\section{(cc) BY}

